\title{
Réflexions du Groupe de travail de la Sociéte française de radioprotection sur l'évolution du système de radioprotection proposée par la CIPR
}

\author{
C. SCHIEBER ${ }^{1}$, Y.-S. CORDOLIANI ${ }^{1}$
}

(Manuscrit reçu le ler octobre 2002, accepté le 25 octobre 2002)

RÉSUMÉ Cet article présente les premières réflexions du Groupe de travail, créé en septembre 2001 par la Société française de radioprotection (SFRP) sur des questions clés qui seront abordées par la Commission internationale de radioprotection (CIPR) lors de l'élaboration de nouvelles recommandations sur la radioprotection. Le choix des sujets considérés résulte de l'analyse du memorandum de la CIPR, publié dans le Journal of Radiological Protection en juin 2001 et de l'expérience des membres du Groupe de travail. Ce sont :

- des réflexions générales sur le nouveau système de protection proposé par la CIPR, - les dimensions individuelle et collective du risque radiologique,

- la comparaison avec la gestion des risques chimiques,

- la protection radiologique de l'environnement,

- les unités dosimétriques.

ABSTRACT Reflections of the Working Group of the French Society for Radiation Protection on key issues proposed by the ICRP.

This paper presents the first reflections of the Working Group which was created by the French Society for Radiation Protection (SFRP) on key issues which will be addressed by the International Commission on Radiological Protection (ICRP) when developing new recommendations on radiological protection. Based on the analysis of the ICRP Memorandum published in the Journal of Radiological Protection in June 2001, and on the experience of the members, the following topics are being considered:

- general reflections on the new system proposed by the ICRP,

- considerations on individual and collective dimensions of risk management,

- comparison with the management of chemical risks,

- radiological protection of the environment,

- dosimetric units.

\section{Introduction}

Répondant à l'invitation de l'Association internationale de protection radiologique (IRPA) en 1999 à participer au débat sur l'évolution du système de protection

\footnotetext{
1 Au nom du Groupe de travail de la SFRP.
} 
radiologique proposée par la Commission internationale de protection radiologique (CIPR), le Conseil d'administration de la Société française de radioprotection (SFRP) a créé un groupe de travail spécifique. Ce groupe s'est réuni d'avril 1999 à juillet 2000, et les résultats de ses travaux ont été présentés lors du congrès IRPA-10 en mai 2000, puis publiés dans la revue Radioprotection (Lecomte et Schieber, 2001a) ainsi que dans le Journal of Radiological Protection (Lecomte et Schieber, 2001b).

En vue de poursuivre les réflexions, le Conseil d'administration de la SFRP a décidé de créer un deuxième groupe de travail (GT) qui a débuté ses travaux en septembre 2001. Ce GT est composé d'une vingtaine de membres représentant les parties prenantes de la radioprotection en France : autorités, experts et professionnels des domaines nucléaire, médical et de la recherche, ainsi qu'un représentant du mouvement associatif (voir liste des membres en fin d'article). L'objectif de ce GT est de formuler des propositions sur les questions clés qui seront abordées par la CIPR, dans le développement de nouvelles recommandations sur la radioprotection. Le choix des principaux sujets débattus résulte de l'analyse du memorandum de la CIPR publié dans le Journal of Radiological Protection en juin 2001 (ICRP, 2001) et de l'expérience des membres du GT. Les thèmes suivants ont ainsi été abordés :

- réflexions générales sur le nouveau système de protection proposé par la CIPR,

- les dimensions individuelle et collective du risque radiologique,

- la comparaison avec la gestion des risques chimiques,

- la protection radiologique de l'environnement,

- la clarification des unités dosimétriques.

Cet article, approuvé par le Conseil d'administration de la SFRP, présente les principales orientations du GT. Il a été élaboré de façon à refléter les diverses opinions qui ont pu être exprimées lors des séances de travail.

\section{Réflexions générales sur le nouveau système de protection proposé par la CIPR}

\subsection{Réflexions sur les principales modifications apportées au système de protection}

Le nouveau système envisagé par la CIPR dans sa publication de juin 2001 (ICRP, 2001 ) est centré sur la protection de l'individu et fondé sur deux considérations s'appliquant à des sources déjà justifiées (la justification des activités étant 
considérée par la CIPR comme ne relevant plus du futur système de radioprotection) :

- la première considération concerne la maîtrise de la dose individuelle. À cette fin, la CIPR propose une classification des doses individuelles annuelles, par niveau croissant d'exposition, appréciées en fonction de leur écart à l'irradiation naturelle de référence (niveau de 1 à $10 \mathrm{mSv}$ par an, qualifié de normal). Cette classification est elle-même complétée par des propositions d'actions de protection. Les types d'action proposés diffèrent selon le type de source - optionnelle ou inévitable - et selon le type d'exposition - public, médical, travailleurs - ;

- la deuxième considération est celle de l'optimisation de la radioprotection. Cette considération est présentée comme relevant d'une exigence morale consécutive à l'adoption de la relation linéaire sans seuil. L'objectif est de diminuer le nombre de personnes exposées et les doses individuelles à un niveau aussi bas que raisonnablement «praticable». Le processus d'optimisation doit en particulier donner lieu à une plus grande implication des acteurs dans l'évaluation et la gestion du risque.

Il apparaît à plusieurs membres du groupe, que ce système, qui, en résumé, propose «de s'intéresser à l'individu en tenant compte du collectif » n'est pas fondamentalement différent du système actuel, dans le sens où il consiste essentiellement à inverser les priorités. Le système actuel, bien que centré sur la dimension collective comporte en effet de nombreuses dispositions permettant de tenir compte de la dimension individuelle.

Il semble important à plusieurs membres du GT de ne pas hiérarchiser les trois principes (justification, optimisation et limitation) mais de les conserver tous, au même niveau d'importance dans le système de protection.

En particulier, plusieurs membres du GT soulignent l'importance de conserver la justification au sein du système de radioprotection, tout en admettant que la décision de justification ne dépend généralement pas des seules considérations de radioprotection. Certains membres pensent également qu'il serait nécessaire, dans le domaine non médical, de s'inspirer de ce qui est préconisé dans le domaine médical. Il s'agirait ainsi de distinguer plusieurs niveaux de justification, notamment :

1. justification de l'utilisation des rayonnements à une fin donnée,

2. justification de l'installation dans laquelle ont lieu des expositions,

3. justification de l'opération conduisant à une exposition,

4. justification de l'exposition d'une personne donnée.

Les points 3 et 4 devraient être intégrés explicitement dans la démarche d'optimisation. 
La proposition de la CIPR d'exclure a priori du système de radioprotection toutes les situations donnant lieu à des expositions individuelles annuelles inférieures à $0,01 \mathrm{mSv}$ est source de débat au sein du GT.

- L'ensemble des membres du GT pense qu'il n'est pas souhaitable de s'affranchir a priori de tout contrôle.

- Certains membres du GT considèrent qu'il est important de replacer la dose dans son contexte avant toute décision relative à sa gestion. Par exemple, les rejets des installations nucléaires, même s'ils donnent lieu à des expositions inférieures à $0,01 \mathrm{mSv}$ par an, ne doivent pas être exclus du système de protection car cela risque de relâcher les contraintes liées à la surveillance de ces rejets. Une exclusion a priori serait antinomique avec le principe d'optimisation selon lequel toute dose, aussi petite soit-elle, doit être évitée dans la mesure du raisonnablement possible. Ceci étant dit, le fait de n'exclure aucun niveau d'exposition du système n'empêche pas de s'arrêter dans la réduction des doses, notamment pour des raisons d'allocations de ressources de protection. Mais cette décision dépend de la situation d'exposition (contexte, ressources nécessaires, ...). Elle est prise au cas par cas dans le cadre de la mise en ceuvre du principe d'optimisation et de la concertation avec les acteurs concernés.

- Pour d'autres membres du GT, notamment issus du monde médical, le fait d'avoir un niveau d'exclusion générique est souhaitable car il permettrait d'éviter a priori les mauvaises allocations de ressources de protection, notamment pour la réduction d'expositions considérées comme non significatives pour le risque individuel qui leur est associé.

- D'autres membres du GT suggèrent de moduler la proposition de la CIPR en donnant un niveau d'exclusion générique à $0,01 \mathrm{mSv}$ par an sauf pour les sources dont le niveau de stabilité ne peut être garanti, en particulier les rejets.

Certains membres du GT, s'interrogent sur la nécessité et la réelle possibilité de simplifier le système actuel. En effet, le premier GT avait déjà noté la diversité et la multiplicité des situations d'exposition ainsi que l'importance d'adapter le système en fonction de ces situations (Lecomte et Schieber, 2001a). Il avait également conclu qu'il serait utile de se doter d'outils permettant de mieux comprendre la démarche adoptée pour la gestion des situations d'expositions. En adaptant le système proposé par la CIPR, il pourrait ainsi être intéressant d'avoir un système qui propose deux considérations séparées.

\section{Première considération : "Appréciation du risque sanitaire individuel »}

Il s'agit ici de s'attacher à l'appréciation du niveau de risque sanitaire associé à une exposition individuelle donnée, quel que soit le contexte d'exposition. Le point de 
départ réside dans deux questions :

(i) quel est le niveau de dose individuelle (sans préoccupation de la source : professionnelle/médicale/public, naturelle/artificielle, réelle/potentielle) ?

(ii) et ce niveau est-il préoccupant d'un point de vue sanitaire?

La dose individuelle pourrait alors être appréciée sur une échelle indiquant le niveau de préoccupation associé à chaque plage de dose individuelle (trois grandes plages de doses individuelles seraient suffisantes). Cette échelle indiquerait également s'il y a lieu de mettre en ouvre une « Action de protection sanitaire ». En considérant trois plages de doses individuelles annuelles, ces actions seraient qualifiées de :

- nécessaire (pour les fortes doses),

- à étudier (pour les doses moyennes),

- ou non pertinente (pour les très faibles niveaux d'exposition).

La nécessité de telles actions serait appréciée ici uniquement en regard des préoccupations liées au risque sanitaire. Il s'agirait d'actions donnant lieu à une obligation de résultats en termes de diminution des expositions individuelles.

\section{Deuxième considération : «Gestion des situations d'exposition »}

Il s'agit ici de considérer l'exposition dans son contexte (i.e. la situation d'exposition déterminée par le type de source, la catégorie de personne exposée, le nombre de personnes exposées, ...), pour déterminer le type d'action de radioprotection que l'on peut envisager pour gérer les expositions individuelles et le nombre de personnes exposées. Pour gérer les différentes situations d'exposition, plusieurs types d'outils ou d'actions sont disponibles (par exemple : limites et contraintes de dose, niveaux de référence, optimisation, niveaux d'intervention, organisation du travail, ...). Les actions engagées pour gérer les différentes situations d'expositions ne relèvent pas ici des seules préoccupations sanitaires, mais intègrent d'autres facteurs de gestion des situations à risque, dont des critères d'acceptabilité et de justification des activités à l'origine de l'exposition. Il devient alors possible de construire un tableau, donnant les types d'actions préconisées selon des familles de situations d'exposition comparables. Il n'existerait pas de lien automatique entre les niveaux d'exposition individuelle de l'échelle de risque individuel présentée dans la première considération, et la classification des situations d'exposition.

L'intérêt de la première considération est de disposer d'une échelle de risque individuel permettant à la personne exposée de situer son exposition et de mettre en perspective le risque encouru vis-à-vis d'autres risques. Une telle échelle serait notamment utile à des fins de communication sur les niveaux de risque ainsi que 
dans le cadre de la constitution progressive d'une culture du risque au sein de la société. La deuxième considération reprend une bonne part du système actuel. II serait important de maintenir une séparation nette entre les deux considérations, tout en notant que l'une ne va pas sans l'autre.

L'ensemble du GT adhère à la proposition de mettre en place une telle échelle de risque individuel, bien que les valeurs de doses individuelles de chaque classe considérée restent encore sujet de débat au sein du GT.

\subsection{Quelques points de terminologie}

La proposition de la CIPR introduit une nouvelle terminologie qui, de l'avis des membres du GT, nuit à la bonne compréhension du système proposé.

- La nouvelle définition de la source, qui distingue source "optionnelle » et source "inévitable».

Le terme «optionnelle » est considéré comme ambigu par certains des membres du GT, car normalement, le système proposé s'applique à des sources qui sont déjà justifiées. Le choix, sous-entendu derrière le terme «optionnelle», est donc supposé être déjà effectué.

D’une façon générale, certains membres du GT souhaiteraient une définition précise du terme de source. En particulier, il conviendrait de définir si une source est définie en fonction de la cible (type d'individu exposé) ou de l'origine des rayonnements (matériel, installation, ...) sur laquelle on peut agir.

- La qualification de niveau "normal " pour les expositions individuelles correspondant au niveau d'irradiation naturel.

Certains membres du GT proposent de remplacer cette qualification par « niveau naturel » ou « niveau moyen ».

- L'indication «pas d'actions de protection» pour les actions de protection typiques associées à certains niveaux d'exposition.

De l'avis de la plupart des membres du GT, cette terminologie est source de malentendus car elle donne à penser que l'on n'applique aucune mesure de radioprotection, de quelque type que ce soit, même pour des doses supérieures au niveau qualifié de négligeable. Si un tel tableau d'actions de protection typiques devait être maintenu, il conviendrait d'indiquer, pour chaque situation d'exposition et selon le niveau d'exposition, s'il y a lieu d'appliquer une action de protection spécifique, de maintenir un processus d'optimisation ou de surveillance voire de s'abstenir d'une action de protection dans la plage du négligeable. 
- La distinction entre ALARA et ALARP.

Le fait de dénommer le principe d'optimisation «ALARP » au lieu de «ALARA » est compris par certains membres du GT comme une volonté d'afficher que l'optimisation de la radioprotection ne se limite pas à une équation coût-bénéfice, mais inclut d'autres considérations et jugements de valeurs.

Certains membres du GT considèrent que, dans la pratique, l'application du principe ALARA fait peu appel à l'équation coût-bénéfice et inclut déjà les autres considérations. Il leur semble que le changement de terminologie serait source de confusion.

\section{Les dimensions individuelle et collective du risque radiologique}

\subsection{La prise en compte de la sensibilité individuelle dans la détermination des niveaux d'action de protection}

La nouvelle orientation proposée pour le système de protection est centrée sur la protection de l'individu. Sachant qu'il existe des différences de sensibilité aux effets des rayonnements selon les individus, la question de la prise en compte de cette sensibilité dans le nouveau système se pose. Bien que cet aspect n'ait pas été traité explicitement dans la proposition de la CIPR, le GT a estimé qu'il était important d'aborder cette question.

Le GT estime qu'il est nécessaire de distinguer deux types de sensibilité :

- une sensibilité « de groupe», fonction de l'âge et du sexe des personnes exposées,

une sensibilité « individuelle », relevant des caractéristiques génétiques des individus.

En ce qui concerne la sensibilité de groupe, le GT considère que les limites d'exposition retenues par la CIPR dans le but de protéger la population dans son ensemble et les travailleurs, sont établies pour protéger une population « moyenne », tenant compte des différences potentielles. Ainsi, par exemple, les limites spécifiques d'exposition adoptées pour les femmes enceintes sont-elles établies pour protéger le fotus. La sensibilité de groupe est donc déjà prise en compte dans le système actuel.

Dans l'optique d'établir un système de protection centré sur l'individu, il apparaît difficile de distinguer trop de groupes d'individus (avec, par exemple, 
des limites annuelles d'exposition différentes suivant l'âge). Le GT estime que le seul domaine d'application du système de radioprotection où il convient de prendre en compte la sensibilité de groupe est le domaine médical. Pour le radiodiagnostic, il est maintenant reconnu que trois types de populations doivent être distingués lors de la réalisation des examens: les enfants, les femmes enceintes et « les autres ». Il semble difficile aux membres du GT de pouvoir aller plus loin dans cette distinction. Pour la radiothérapie, l'adaptation du traitement se fait au cas par cas, en fonction des réactions initiales de l'individu traité.

En ce qui concerne la sensibilité individuelle, le GT remarque que la possibilité de tester la sensibilité génétique des individus donne lieu à des recherches scientifiques mais n'est pas opérationnelle à grande échelle. Si une telle possibilité devait voir le jour, le GT estime qu'il serait nécessaire de mener un débat de fond sur les aspects éthiques et sociaux liés à l'utilisation de ces tests, en particulier, dans le cas de l'exposition de travailleurs.

En conclusion, dans l'optique d'une orientation du système de protection vers l'individu, le GT estime qu'il est essentiel que le nouveau système prenne en compte la radiosensibilité de groupe et indique les modalités de cette prise en compte. En ce qui concerne la radiosensibilité individuelle, le GT invite la CIPR à entreprendre dès à présent une réflexion sur les limites éthiques et sociales qu'il conviendrait de considérer. Étant donné que ce sujet déborde du champ de la radioprotection, il conviendrait d'associer d'autres parties prenantes à cette réflexion.

\subsection{L'utilisation de la dose collective}

Le GT a mené une réflexion sur l'utilisation de la dose collective sur la base d'un document produit par un groupe de travail de l'Institut de protection et de sûreté nucléaire (IPSN, 2002) et repris à son compte par l'Institut de radioprotection et de sûreté nucléaire (IRSN).

En résumé, l'IRSN considère la dose collective comme un indicateur utile, bien que non exclusif, de la dimension collective du risque, si cet indicateur est utilisé à bon escient en tenant compte de certaines limites. La dose collective peut être utilisée pour évaluer l'impact dosimétrique d'une activité ou d'une source sur un groupe de personnes, ou pour apprécier le nombre d'effets stochastiques qui pourraient résulter de l'exposition d'un groupe de personnes. Les méthodes de calcul de la dose collective et son utilisation doivent être adaptées à la nature de la situation considérée. Les principales limites à apporter lors de 
l'utilisation de la dose collective présentées dans le document de 1'IRSN sont les suivantes.

- Il importe de ne pas amalgamer des situations et des conditions d'exposition disparates. Il convient par conséquent de calculer la dose collective pour des segments homogènes de telle sorte que chaque segment regroupe des doses individuelles similaires (pas de différences supérieures à un ou deux ordres de grandeur au maximum), que les périodes de temps ne diffèrent pas de plus d'un ou deux ordres de grandeur, et que les doses très faibles ne sont pas négligées a priori. La démarche de segmentation et d'appréciation des segments constitue une démarche plus réaliste et plus crédible que le recours à une dose « de minimis » générique.

- Quand la dose collective est calculée en vue d'une estimation de risque, la segmentation doit s'attacher à ne pas regrouper des doses individuelles présentant des risques différents, à ne pas considérer des périodes supérieures à quelques dizaines d'années (maximum un siècle), et enfin à considérer uniquement des groupes de population présentant des conditions biologiques identiques (âge par exemple) en raison notamment de radiosensibilités différentes.

- Enfin, avant toute interprétation des résultats d'un calcul de dose collective, il est indispensable de connaître les principaux composants de cette dose (nombre de personnes exposées, niveau moyen d'exposition individuelle, distribution des doses individuelles, ...).

Dans son ensemble, le GT adhère aux propositions du document de l'IRSN à quelques nuances près :

- tous les membres du GT sont d'accord pour considérer que la dose collective est un indicateur de performance utile. Elle constitue notamment un bon outil opérationnel pour la radioprotection des travailleurs. Elle est, entre autres, nécessaire lors de l'application de la démarche d'optimisation de la radioprotection ;

- en revanche, certains membres du GT expriment leur opposition à utiliser la dose collective comme indicateur de risque dans une population donnée, par exemple pour calculer un nombre d'effets potentiels dans une population, notamment dans le domaine des irradiations médicales en radiodiagnostic.

La question de la fixation ou non d'un niveau « de minimis » de dose individuelle en dessous duquel il ne faudrait pas réaliser de calcul de dose collective est également sujet de débat au sein du GT.

- Pour certains membres du GT, il conviendrait de fixer un niveau « de minimis » a priori, afin de ne pas considérer les très faibles doses dans le calcul de la dose collective. Ce niveau pourrait, par exemple, être de l'ordre de grandeur de la fluctuation de la radioactivité naturelle. 
- Pour d'autres membres du GT, le niveau «de minimis » générique est impossible à déterminer, mais doit être fixé au cas par cas.

- D'autres membres enfin, sont de l'avis de l'IRSN qui considère qu'il convient, selon les situations et la question posée, de ne pas s'affranchir systématiquement du calcul de la dose collective même pour les très faibles doses. Ils estiment notamment que l'intervalle de fluctuation de la radioactivité naturelle ne constitue pas un seuil d'innocuité des effets potentiels des rayonnements.

\section{Mise en perspective avec la gestion des risques chimiques}

Il est apparu important aux membres du GT de mener une première réflexion permettant d'expliciter les différences éventuelles entre les modes d'évaluation et de gestion des risques chimiques cancérogènes et des risques radiologiques, en vue de s'interroger sur les possibilités de rapprochement des modalités de gestion de ces risques.

Les réflexions ont été menées autour de trois principaux thèmes :

- les modes d'évaluation des risques chimiques cancérogènes,

- les modalités de gestion des risques chimiques,

- la prise en compte des situations à risques multiples.

\section{Évaluation des risques chimiques cancérogènes}

Dans le cas particulier des substances cancérogènes dites «génotoxiques », il est intéressant d'observer que les organismes chargés de l'évaluation des risques chimiques dans divers pays (OMS, US-EPA, ...) adoptent l'hypothèse prudente d'une relation exposition-risque « linéaire sans seuil », à l'instar de ce qui est fait dans le domaine du risque radiologique. Le statut de ces relations linéaires sans seuil est important : il s'agit de modèles simplifiés utilisés dans le cadre de la gestion des risques et non des modèles censés refléter les mécanismes biologiques. De même que dans le domaine du risque radiologique, certaines controverses émergent sur l'existence ou non d'un seuil, mais d'une façon générale, la quantification du risque cancérogène aux faibles doses est réalisée à l'aide des relations linéaires sans seuil.

\section{Modalités de gestion des risques cancérogènes chimiques}

En ce qui concerne la protection du public, les risques associés aux substances cancérogènes chimiques sont généralement gérés par l'adoption de valeurs limites fixées en considérant d'une part un critère de risque sanitaire, et, d'autre part, un critère de faisabilité fondé sur les meilleures technologies de protection disponibles. En ce qui concerne le risque sanitaire, l'OMS propose, par exemple, 
des valeurs guides pour la qualité de l'air et pour la qualité de l'eau, qui correspondent à un risque de cancer pour le public de $10^{-5}$ sur la vie entière pour une exposition pendant 70 ans. Les différents organismes en charge de la fixation des valeurs limites utilisent le plus souvent les valeurs guides de l'OMS en les adaptant si nécessaire, en fonction des technologies disponibles.

Il est à noter que la notion de risque négligeable est utilisée par certains organismes lors de la détermination des valeurs guides ou des valeurs limites pour les cancérogènes chimiques. Aux États-Unis, la valeur utilisée par l'EPA est de $10^{-6}$ risque d'apparition d'un excès de cancer (et non de décès par cancer) sur la vie entière, alors que le HSE au Royaume-Uni se réfère à la valeur de $10^{-6}$ par an .

Le principe d'optimisation de la protection n'existe pas en tant que tel dans les politiques de gestion du risque chimique. Cependant, il est intéressant de noter que, pour des cas particuliers comme la gestion de la qualité de l'eau, l'US-EPA détermine deux valeurs :

(i) une valeur limite de concentration tenant compte des coûts et de la faisabilité technique,

(ii) et une valeur correspondant à un objectif de concentration dans l'eau, déterminé pour des raisons sanitaires.

Cette dernière valeur est inférieure ou égale à la valeur limite, et détermine ainsi l'objectif à atteindre.

En ce qui concerne la protection de l'environnement, il est admis d'évaluer les risques pour les écosystèmes, associés aux rejets de substances chimiques. Cette démarche est encore en cours d'exploration dans le domaine des risques radiologiques (cf. Sect. 5).

\section{Prise en compte des situations à risques multiples}

Les membres du GT soulignent la nécessité, dans de nombreuses situations, d'arbitrer entre plusieurs types de risques (par exemple radiologiques et chimiques) lors de la recherche des niveaux de risque acceptables. Il serait utile que la CIPR engage une réflexion sur le sujet, en se basant sur des études de cas concrets.

En conclusion, le GT constate que sur certains aspects (notamment en matière d'évaluation du risque), il existe une certaine cohérence entre la gestion des risques cancérogènes chimiques et radiologiques. Cependant, il importe que cette cohérence soit renforcée. Le GT suggère que la CIPR se rapproche des experts en matière de risques cancérogènes chimiques afin de faciliter une harmonisation et une cohérence entre les modalités de gestion de ces deux types de risque. 


\section{La protection radiologique de l'environnement}

La problématique de la protection radiologique de l'environnement s'inscrit dans un contexte international nouveau, exprimé notamment lors des conférences de Rio et de Kyoto, et caractérisé par le développement du concept de développement durable et par l'adoption du principe de précaution.

Depuis quelques années, de nombreux organismes ont commencé à réfléchir sur la protection radiologique de l'environnement. On peut notamment citer le programme de recherche européen FASSET, le programme Envirhom de l'Institut de radioprotection et de sûreté nucléaire (IRSN, France), le Groupe de travail de l'Union internationale de radioécologie (IUR), les réunions d'experts organisées par l'AIEA, ainsi que le récent « task group » créé par la CIPR (Bréchignac, 2001).

Le GT est de l'avis que la position actuelle de la CIPR, telle qu'exprimée par exemple dans la CIPR 60, qui consiste à considérer que la protection de l'homme conduit à protéger l'environnement, répond imparfaitement aux préoccupations actuelles. En particulier, le GT remarque que :

- cette position ne prend pas en compte la protection de l'environnement en tant que tel et en particulier celle des espèces animales et végétales dont le développement est aujourd'hui sans interaction avec celui de l'homme ; ce faisant, elle prend imparfaitement en compte la protection des générations humaines futures ;

- il n'existe pas à ce jour de critères et de normes reconnues au niveau international concernant la qualité de l'environnement ;

- il serait nécessaire de développer au niveau international des méthodes cohérentes de mesure dans l'environnement ;

- la protection de l'homme nécessite une approche "système ", prenant en compte les interactions nombreuses et complexes entre le milieu et les populations vivantes. L'objet de la protection de l'environnement est donc l'écosystème.

Le GT encourage la CIPR à poursuivre ses réflexions concernant la protection radiologique de l'environnement, en veillant à s'entourer de spécialistes de la question, y compris hors du domaine de la radioprotection. Il souligne l'importance de consulter l'ensemble des parties prenantes dans l'élaboration des critères de qualité qui seront nécessaires pour évaluer l'efficacité de la protection radiologique de l'environnement.

\section{Les unités dosimétriques}

Le GT s'est attaché à recenser les grandeurs dosimétriques utilisées dans les différents domaines (patients, travailleurs, public) et leurs unités, en distinguant 
les grandeurs opérationnelles (mesurées) des grandeurs de radioprotection (calculées).

Il est apparu que le principal problème posé actuellement réside dans l'emploi d'une même unité pour exprimer la dose équivalente à l'organe et la dose efficace, ce qui entraîne des confusions importantes. Ce problème est notamment rencontré dans certaines publications du domaine médical qui indiquent, par exemple, les doses reçues par les patients lors d'examens radiologiques en dose équivalente (donc en $\mathrm{mSv}$ ), sans le préciser clairement, ces doses étant par la suite interprétées comme étant des doses efficaces (ou inversement).

Le GT recommande fortement que la même unité ne soit pas utilisée pour les deux grandeurs de dose équivalente et de dose efficace.

Par ailleurs, le GT estime que les publications de la CIPR devraient s'attacher à donner plus de précision sur les différentes grandeurs, et leur correspondance.

Le GT souhaite que, si des modifications doivent être apportées aux coefficients de pondérations $\left(W_{T}\right.$ et $W_{R}$ ), la CIPR tienne compte de l'impact de ces modifications sur la radioprotection en général et le calibrage de certains dosimètres (notamment neutrons) en particulier. Le GT recommande la plus grande cohérence entre les grandeurs de protection définies par la CIPR et les grandeurs opérationnelles définies par l'ICRU.

\section{Remerciements et perspectives}

La Société française de radioprotection remercie la CIPR d'avoir ouvert le débat sur l'évolution du système de protection. Elle remercie également l'IRPA de donner l'occasion aux parties prenantes de présenter leurs réflexions dans un cadre international, propice au dialogue et au partage d'expériences.

Le GT va poursuivre ses travaux. Il souhaite que le prochain grand rendez-vous international de l'IRPA à Madrid en 2004 soit à nouveau l'occasion d'échanger les points de vue des parties prenantes de la radioprotection.

\section{Liste des membres du Groupe de travail}

AUBERT Bernard, IGR; AMMERICH Marc, DGSNR; BARBEY Pierre, ACRO ; BLAIN Alain, FRAMATOME - ANP; BOURGUIGNON Michel, DGSNR ; CORDIER Gérard, EDF/DPN/FTC ; CORDOLIANI Yves-Sébastien, SFR, HIA Val-de-Grâce ; DELIGNE Jean-Michel, IRSN ; DORMONT Dominique, CEA ; FRACAS Patrick, CEA ; GRANET Marie-Béatrice, Université 
de Poitiers; HEMIDY Pierre-Yves, EDF; KIFFEL Thierry, DGSNR ; LACRONIQUE Jean-François, IRSN ; LAURENT Jean-Pierre, COGEMA ; LECOMTE Jean-François, CIPR; LE GUEN Bernard, EDF; LOMBARD Jacques, SFRP; MAKOVICKA Libor, Université de Franche Comté; MÉTIVIER Henri, CIPR ; OUDIZ André, IRSN ; PASQUIER Jean-Luc, IRSN ; PAUL Didier, INSTN ; RENAUD-SALIS Valérie, IRSN ; SCHIEBER Caroline, CEPN.

\section{RÉFÉRENCES}

Bréchignac F. (2001) Impact of radioactivity on the environment: problems, state of the current knowledge and approaches for identification of radioprotection criteria, Radioprotection 36, $511-535$.

ICRP (2001) A report on progress towards next recommendations: a communication from the International Commission on Radiological Protection, J. Radiol. Prot. 21, 113-123.

IPSN (2002) Collective Dose - Indications and contraindications, Document IPSN. EDP Sciences, Les Ulis.

Lecomte J.-F., Schieber C. (2001a) Contribution de la Société française de radioprotection aux réflexions actuelles sur les possibles améliorations du système de gestion du risqué radiologique, Radioprotection 36, I l-40.

Lecomte J.-F., Schieber C. (2001b) Contribution of the French Society for Radiation Protection to the current reflections on the possible improvements to the radiological risk management system, $J$. Radiol. Prot. 21, 277-300. 